\title{
Paediatric orbital alveolar soft part sarcoma recurrence during long-term follow-up: a report of 3 cases and a review of the literature
}

Yujiao Wang, Baixue Du, Mei Yang and Weimin $\mathrm{He}^{*}$

\begin{abstract}
Background: Alveolar soft part sarcoma (ASPS) is a clinically and morphologically distinct malignant soft tissue tumour. It occurs mostly in the lower extremities in adults. The purpose of our study was to describe the related clinicopathologic factors, treatment and prognosis of recurrent orbital ASPS in children.

Case presentation: Three children aged from 1 to 12 years presented with unilateral proptosis, restricted ocular motility and impaired visual acuity of the affected eye. Periodic acid Schiff (PAS) -positive diastase-resistant crystalline granules were found in all cases. Immunostaining of TFE-3, INL1 and Ki67 was positive in the tumour cells of two patients. The time until local recurrence of primary tumor in patient 2 and patient 3, who only underwent tumour resection, was shorter than that of patient 1, who underwent tumour excision and postoperative radiotherapy. The recurrent masses were successfully treated with complete tumour excision followed by adjuvant radiotherapy. Patient 1 presented metastasis at 11 years after radiotherapy.

Conclusions: Orbital ASPS in children is easily misdiagnosed due to its rare occurrence and atypical clinical findings. Early diagnosis with multidisciplinary, complete surgical resection combined with adjuvant radiotherapy is essential for achieving long-term disease-free survival in orbital ASPS patients.
\end{abstract}

Keywords: Alveolar soft part sarcoma, Orbital tumour, Tumour recurrence, Histopathology, Differential diagnosis

\section{Background}

Alveolar soft part sarcoma (ASPS) is an extremely rare soft tissue sarcoma that accounts for $0.5-1 \%$ of all sarcomas [1] and has unique histological features, an uncertain cell of origin and variable clinical behaviour. The tumour mostly occurs in adolescents and young adults, and females are more often affected [2]. It usually involves the muscles and deep soft tissue of the extremities, trunk, head and neck, whereas in children, it primarily affects the head and neck regions [3, 4]. As ASPS is a rare orbital tumour and relapses easily, ophthalmologists and pathologists should be aware of the related clinicopathologic features and the potentially aggressive nature of the disease for the early implementation of available

\footnotetext{
* Correspondence: weimin_he@yeah.net

Department of Ophthalmology, Ophthalmic Laboratory, West China Hospital, Sichuan University, Chengdu 610041, China
}

treatment options. We reported three children with recurrent orbital ASPS and briefly reviewed the literature.

\section{Case presentation}

After review of the diagnoses of cases accessioned in the database of the Ophthalmology Department and Pathology Department of West China Hospital of Sichuan University, three cases of orbital alveolar soft part sarcoma (ASPS) diagnosed between 1993 and 2019 were identified. The clinical histories, manifestations and pathologic findings of the tumours were reviewed. A literature review was performed using MEDLINE for studies published between 1950 and the present, and EMBASE was searched for studies published between 1980 and the present.

(c) The Author(s). 2020 Open Access This article is distributed under the terms of the Creative Commons Attribution 4.0 International License (http://creativecommons.org/licenses/by/4.0/), which permits unrestricted use, distribution, and reproduction in any medium, provided you give appropriate credit to the original author(s) and the source, provide a link to the Creative Commons license, and indicate if changes were made. The Creative Commons Public Domain Dedication waiver (http://creativecommons.org/publicdomain/zero/1.0/) applies to the data made available in this article, unless otherwise stated. 


\section{Case 1}

A 9-year-old Chinese girl sought treatment for a fivemonth history of prominence of the left eye. On examination, the corrected visual acuity was 20/20 OD and 20/ 25 OS. The left eye had a nonpulsatile and nonreducible proptosis of $8 \mathrm{~mm}$, as revealed by a Hertel exophthalmometer. A dilated epibulbar vessel on the temporal aspect of the left eye and dilated retinal veins of the left fundi were noted by indirect ophthalmoscopy. CT imaging indicated a $3.5 \times 2 \times 2.5 \mathrm{~cm}$ well-defined soft tissue mass occupying the posteroinferior part of left orbit in close relation to the optical nerve that did not invade the orbital wall (see Fig. 1. a). Left lateral orbitotomy showed an intraconal mass surrounding the optic nerve and adherent to neighbouring tissue in the temporal part of the inferior orbit. Tumour excision was performed to achieve a negative surgical margin whenever possible, and the success of this was examined histologically at the point closest to the resected specimen. The pathological diagnosis was ASPS. Subsequently, the girl was scheduled for adjuvant radiotherapy. Seven years later, she returned with gradual progressive painless proptosis of the left eye for eight months. On examination, there was a slight limitation of the left gaze and a $4 \mathrm{~mm}$ proptosis of the left eye. CT imaging showed an enhancing retrobulbar tumour of $1.3 \times 1.0 \times 1.0 \mathrm{~cm}$ impinging on the left lateral rectus. The mass was completely resected, and the pathologic diagnosis was recurrent ASPS. The patient underwent regular adjuvant radiotherapy (50 Gy in 15 fraction) for two months. At 7 months after radiotherapy, she did well, with no evidence of metastasis or recurrence. At nearly 11 years, the patient died of cerebral metastases, as reported by telephone follow-up.

\section{Case 2}

A 12-year-old girl presented left upper eyelid swelling and prominence of the left eye for one month. Her corrected vision was 20/20 OD and 20/40 OS. The left eye showed a proptosis of $3 \mathrm{~mm}$ with limited motility in all directions. The remainder of the ophthalmic examination results were normal. CT imaging revealed a hyperdense, well-defined intraconal mass of $2.4 \times 2.0 \times 1.5 \mathrm{~cm}$ that compressed the posterior of the eyeball and the superior part of the optic nerve. Although the mass was resected by left orbitotomy and pathologically confirmed to be ASPS, the patient returned with painless prominence of the left eye two months later, indicating local recurrence. Examination of the left eye showed marked eyelid swelling, chemosis and a proptosis of $4 \mathrm{~mm}$ with limited motility. A CT scan revealed a tumour of $4.2 \times$ $4.0 \times 2.8 \mathrm{~cm}$. After adequate surgical removal tumour (see Fig. 1. b), the pathologic diagnosis of the tumour revealed ASPS recurrence. At that point, the girl underwent regular adjuvant radiotherapy $(60 \mathrm{~Gy}$ in 20 fraction). At 3 years, she was alive, with no evidence of recurrence or metastasis. Subsequently, she was lost to follow-up.

\section{Case 3}

Five months before admission to the hospital, a 1-year-old boy was noticed to have eyelid swelling accompanied by diminished ocular movements of his left eye. On examination, there was a left proptosis of $4 \mathrm{~mm}$ as well as redness and conjunctiva. Ocular motility was restricted on abduction. MRI revealed a hyperdense, extraconal mass of $3.8 \times 2.5 \times$ $1.6 \mathrm{~cm}$ that was located in the posterosuperior part of the left orbit in close proximity to the optic nerve and lateral rectus and that compressed the lateral eyeball (see Fig. 2). The right eye was normal. After locally and completely excising the mass together with the adjacent soft tissue, the histopathologic diagnosis was ASPS. His parents refused post-surgical radiotherapy, and the patient was then lost to follow-up. Eight years later, the parents sought treatment for a five-year history of prominence of the left eye after the first surgery. Our examination showed a left proptosis of $3 \mathrm{~mm}$ with limited ocular motility in all directions. MRI revealed a $4.2 \times$

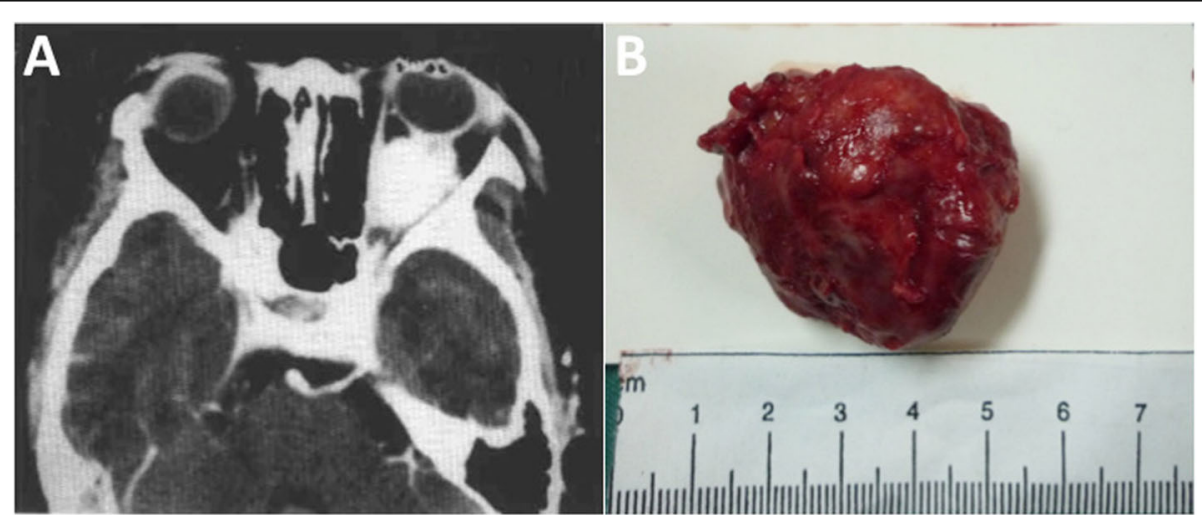

Fig. 1 a Axial CT scan of the orbits in patient 1, showing a heterogeneous mass surrounding the optic nerve in the posteroinferior orbit. $\mathbf{b}$ Left orbital mass resected from patient 2 


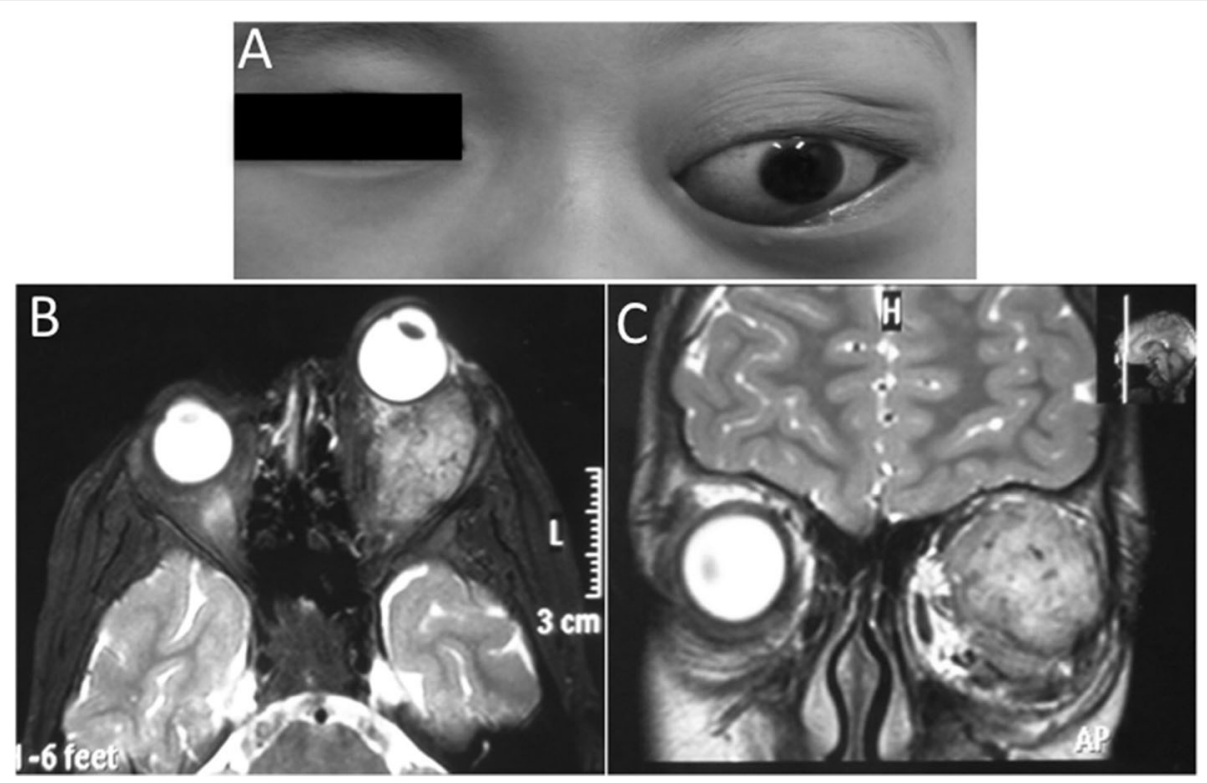

Fig. 2 a Nonaxial proptosis of the left eye in patient 3, with downward displacement of the globe; $\mathbf{b}$ and $\mathbf{c}$, T2-weighted axial and coronal series showing a well-defined recurrent mass with intermediate signal intensity in the posterosuperior orbit

$2.7 \times 3.5 \mathrm{~cm}$ retro-ocular mass. The tumour was excised by left orbitotomy, and the pathologic diagnosis indicated ASPS recurrence. The patient was scheduled for (60 Gy in 20 fraction) adjuvant radiotherapy in light of the close surgical margins. He remains under follow-up and is free of recurrence or metastasis.

\section{Pathologic findings}

Gross specimens of the tumour appeared yellow to brown in patient 1 and red brown in both patient 2 and patient 3 . The specimens were all irregularly shaped soft to firm masses. Microscopic examination of the primary and recurrent tumours from three cases showed that the

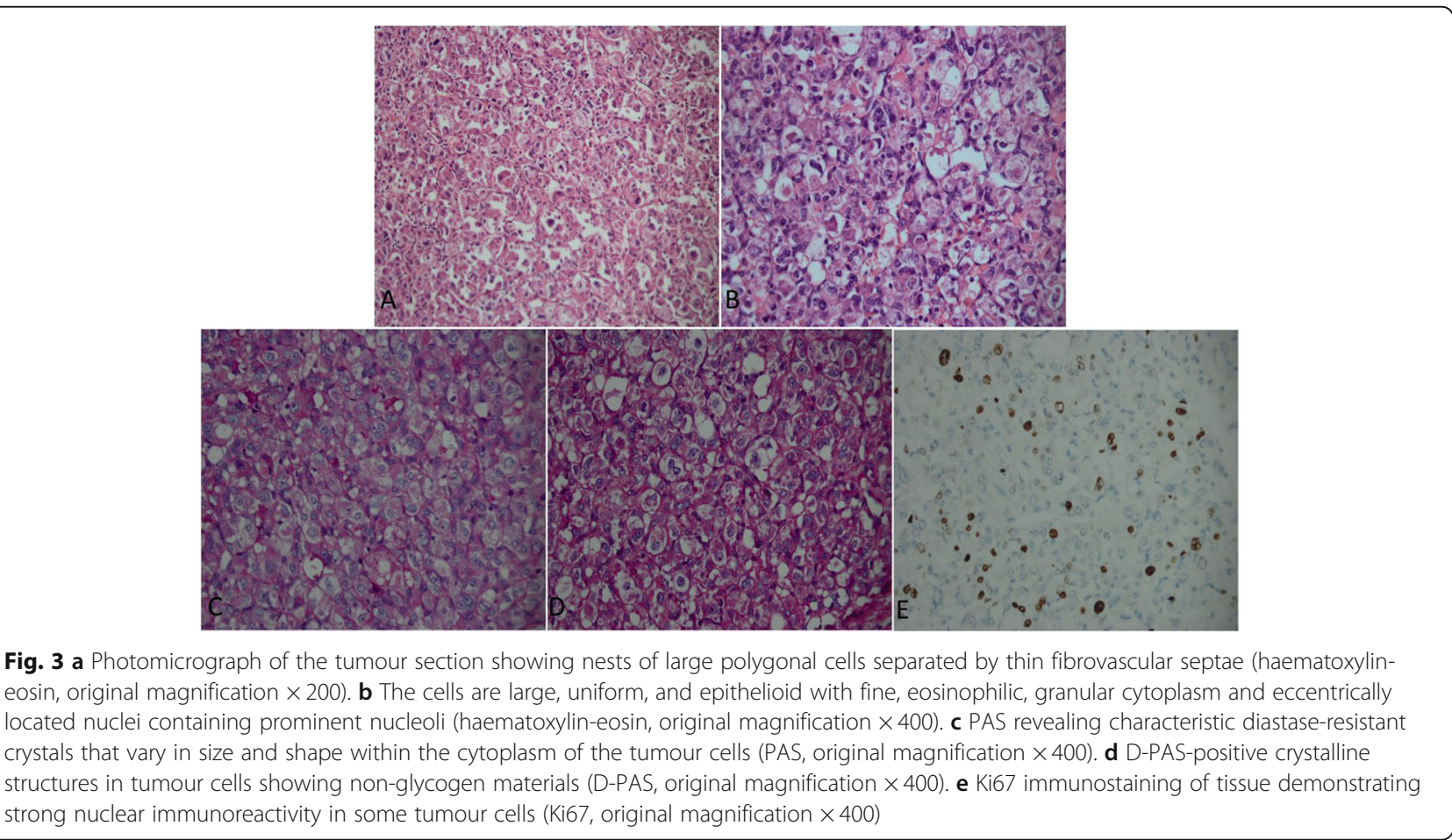


large oval or polygonal tumour cells were arranged in an organoid or alveolar pattern separated by delicate septa of connective tissue containing capillary-sized vascular channels (Fig. 3. a). The tumour cells had a distinctly epithelioid appearance with well-defined cell borders, abundant eosinophilic cytoplasm and large vesicular nuclei with prominent nucleoli (Fig. 3. b). Periodic acid Schiff (PAS) staining revealed characteristic diastaseresistant crystalline structures that varied in size and shape within the cytoplasm (Fig. 3. c-d). Nuclear atypia and mitotic figures were seen in the tumour cells of the recurrent ASPS in patient 2. Muscle markers (desmin, vimentin, myoglobin, myogenin, MyoD1, and SMA) and non-muscle markers (S-100, TFE-3, INL1, CD34, CD31, EMA and Ki67) were used for immunohistochemical staining in our study (Fig. 3-4). The tumours from patient 1 were positive for S100 and myoglobin. TFE-3, INL1 and Ki67 stained positive in patient 2. The tumours of patient 3 were positive for TFE-3, INL-1, CD34, Desmin, and Ki67(ranging from 10 to $20 \%$ positivity), indicating the proliferative potential of ASPS.

\section{Discussion and conclusions}

ASPS is a malignant soft tissue sarcoma that rarely occurs in the orbit. A review of the literature indicates 60 reported cases in the orbit to date [4-29]. Orbital ASPS occurs in young patients between 15 and 35 years and has a 3.25:1 female preponderance.
The location of ASPS in children is more common in the head and neck regions, especially in the orbit. The cases of orbital ASPS we reported here all occurred in children less than 12 years old, including two girls and a boy.

ASPS usually presents as a slow-growing, painless tumour mass. Orbital lesions have no typical characteristics in terms of clinical and imaging features. ASPS commonly presents proptosis, lid swelling, and dilation of conjunctival vessels. Some patients show compression of the optic nerve, as was seen in our cases, which might lead to impaired vision. PAS staining reveals the characteristic diastase-resistant crystalline structures in ASPS, and D-PAS staining was also used to demonstrate that the PAS-positive structures were not glycogen. Although orbital ASPS has characteristic histopathology, its immunohistochemical staining pattern has been controversial, and the histogenesis of ASPS remains uncertain. Some muscle markers (desmin, vimentin, myoglobin, and MyoD1) and non-muscle markers (S-100) have been reported to be expressed at variable frequencies in ASPS. Our report showed that TFE-3 and INL1 were positive in both the primary and recurrent tumours of two patients. The current concepts demonstrate that ASPS is characterized by the translocation $t(X ; 17) \mathrm{p}(11.2 ; \mathrm{q} 25)$, which results in the chimeric ASPSCR1-TFE3 transcription factor, which drives tumorigenesis and provides an important clue for the diagnosis of ASPS [30, 31].

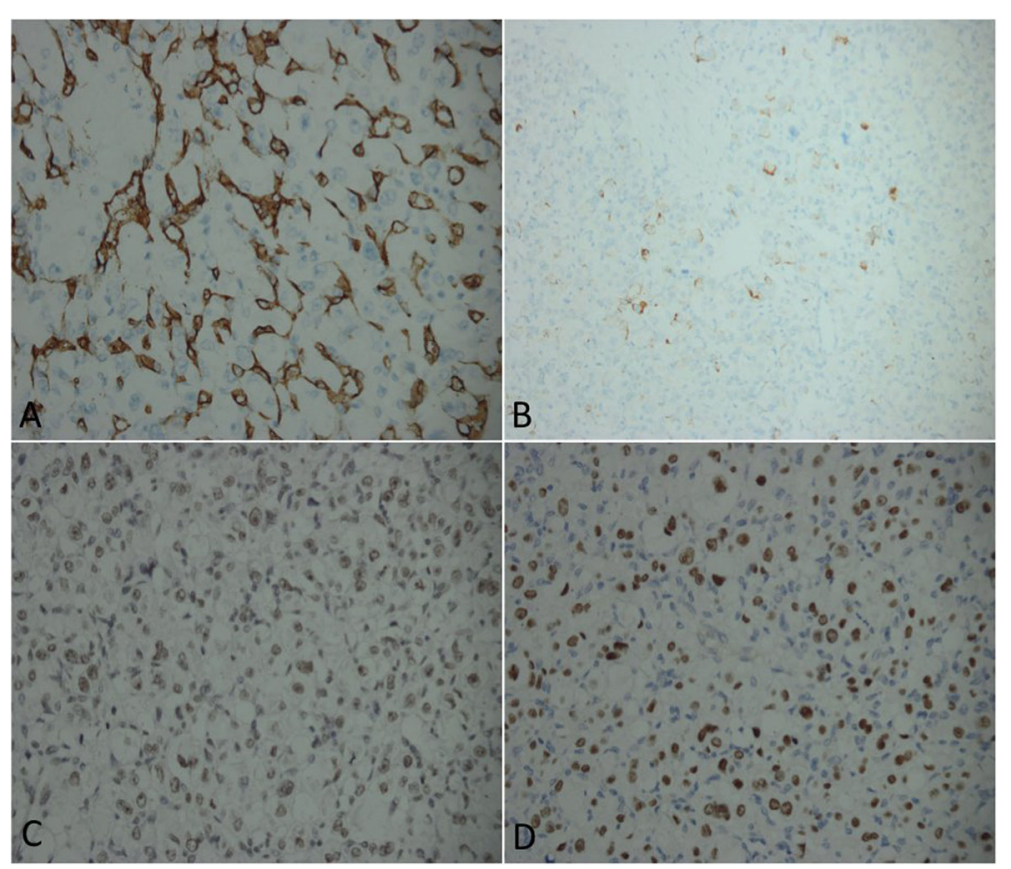

Fig. 4 a CD34 immunostaining of specimens showing diffuse cytoplasmic immunoreactivity in tumour cells (CD34, original magnification $\times 400)$. b Desmin immunostaining of tumour cells revealing cytoplasmic positivity (Desmin, original magnification $\times 400$ ). $\mathbf{c}$ INL-1 staining of tumour cells showing nuclear immunoreactivity (INL-1, original magnification $\times 400)$. d Some tumour cells of the specimen show nuclear immunoreactivity after TFE-3 staining (TFE-3, original magnification $\times 400$ ) 
ASPS may be treated as a vasogenic tumour due to its high vascularization on CT imaging, including haemangioma or lymphangioma. The pathologic differential diagnoses of ASPS include paraganglioma, malignant melanoma, alveolar rhabdomyosarcoma, granular cell tumours and metastatic renal cell carcinoma (RCC), which also need to be considered [32]. ASPS usually presents prominent large nucleoli, which are not seen in paraganglioma, which tends to have striking anisonucleosis. Melanoma cells have a better preserved cytoplasm with pigmentation, a clear vacuolated cytoplasm is common in RCC, and alveolar rhabdomyosarcoma cells have a larger nucleus with atypical features and mitotic figures, as compared to ASPS [ 7, 32]. In addition, ASPS is usually negative for epithelial markers (EMA and CK), neuroendocrine markers (synaptophysin and chromogranin A) and melanocytic markers (HMB45 and melan A).

Due to the rare occurrence of orbital ASPS, the optimal management remains controversial. Although complete surgical excision of the small tumour with functional preservation remains the treatment of choice [33], it is difficult to achieve negative surgical margins due to the poorly circumscribed nature of the tumour and its adherence to critical structures such as the optic nerve or extraocular muscles. Local radiotherapy may be beneficial for ASPS by enhancing the local control achieved with limited surgery, by retarding the progression of metastatic deposits, and by providing meaningful palliation [33-35]. Our cases also included small tumours with diameters of less than $5 \mathrm{~cm}$. We performed complete tumour excision followed by local radiotherapy (50-60 Gy in 15-20 fraction) to achieve improved local control and preserved vision. Although the most commonly used chemotherapy agents, such as doxorubicin and ifosfamide, were used in metastatic cases, none have proven to be beneficial $[34,36]$. The role of neoadjuvant chemotherapy is also unclear.

The prognosis of ASPS is largely dependent on the initial presentation (localized or metastatic disease), tumour size, and age of onset (patients less than 20 years old may survive significantly longer than those over 20 years old) [37, 38]. Orbital ASPS shows a high incidence of local recurrence in 18.6 to $47.7 \%$ of cases. Tumour metastasis might occur late in the course of the disease (median 6 years) and usually appears in the lung, brain, or bone $[27,37,38]$. Interestingly, the two patients that only underwent primary tumour resection presented local recurrence at nearly 2 months and 3 years. Patient 1 had tumour recurrence at 7 years after postoperative radiotherapy. All recurrent masses were successfully treated with tumour resection followed by adjuvant radiotherapy. Patient 1 presented brain metastasis at 11 years after adjuvant radiotherapy. Thus, complete tumour resection and postoperative radiotherapy may offer a better strategy for improving local control. However, we also need more studies to prove the effects of postoperative adjuvant radiotherapy in ASPS. In addition, long-term follow-up is also necessary, as distant metastasis can occur.

\section{Abbreviations}

ASPS: Alveolar soft part sarcoma; CT: Computed tomography; MRI: Magnetic Resonance Imaging

\section{Acknowledgements \\ Not applicable.}

Authors' contributions

Conceptualization of the study: WMH. Data acquisition: YJW and BXD. Manuscript preparation: YJW and MY. Revision of manuscript: WMH and YJW. All authors have read and approved the final manuscript.

\section{Funding}

This study was supported by a grant from the 1.3.5 Project for disciplines of excellence-Clinical Research incubation Project, West China Hospital, Sichuan University (2018HXFH024) and a grant from Post-Doctor Research Project, West China Hospital, Sichuan University (2019HXBH051). West China Hospital, Sichuan University is the only funder of our study and had no competing interests.

\section{Availability of data and materials}

The datasets used and/or analyzed during the current study are available from the corresponding author on reasonable request.

\section{Ethics approval and consent to participate}

This study complied with the tenets of the Declaration of Helsinki. IRB approval was exempted because this was only case report.

\section{Consent for publication}

Parental consent was obtained from the patients' parents for publication of the case report and accompanying images. Written informed consent for publication of the clinical details was obtained from the patients' parents.

\section{Competing interests}

The authors declare that they have no competing interests.

Received: 9 August 2019 Accepted: 8 January 2020

Published online: 21 February 2020

\section{References}

1. Christopherson WM, Foote FW Jr, Stewart FW. Alveolar soft part sarcomas. Structurally characteristic tumours of uncertain histiogenesis. Cancer. 1952;5: 100-11.

2. Bunt $A H$, Bensinger RE. Alveolar soft part sarcoma of the orbit. Ophthalmology. 1981;88:1339-46.

3. Altamirano-Dimas M, Albores-Saavedra J. Alveolar soft part sarcoma of the orbit. Arch Ophthalmol. 1966;75:496-9.

4. Font RL, Jurco S 3rd, Zimmerman LE. Alveolar soft-part sarcoma of the orbit: a clinicopathologic analysis of seventeen cases and a review of the literature. Hum Pathol. 1982;13:569-79.

5. Coupland SE, Heimann H, Hoffmeister B, et al. Immunohistochemical examination of orbital soft part sarcoma. Graefes Arch Clin Exp Ophthalmol. 1999;237:266-72.

6. Archer KF, Hurwitz JJ, Balogh JM, et al. Orbital nonchromaffin paraganglioma. A case report and review of literature. Ophthalmology. 1989:96:1659-66.

7. Ordóñez NG, Mackay B. Alveolar soft- part sarcoma: a review of the pathology and histogenesis. Ultrastruct Pathol. 1998;22(4):275-92.

8. Nirankari MS, Greer $\mathrm{CH}$, Chaddah MR. Malignant non-chromafine paraganglioma in the orbit. Br J Ophthalmol. 1963;47:357-63.

9. Abrahams IW, Fenton RH, Vidone R. Alveolar soft part sarcoma of the orbit. Arch Ophthalmol. 1968;79:185-8.

10. Goder G. Das nichtchromafine paragangliom der orbita. Zbl Allg Path Bd. 1970;113:167. 
11. González-Almaraz G, de Buen S, Tsutsumi V. Granular cell tumor (myoblastoma) of the orbit. Am J Ophthalmol. 1975;79:606-12.

12. Mukherjee PK, Agrawal S. Alveolar soft part sarcoma of the orbit. Indian J Ophthalmol. 1979;27:15-7.

13. Grant GD, Shields JA, Flanagan JC, et al. The ultrasonographic and radiologic features of a histologically proven case of alveolar softpart sarcoma of the orbit. Am J Ophthalmol. 1979;87:773-7.

14. Chodankar CM, Pandit SP, Joshi MG, et al. Alveolar soft-part sarcoma of the orbit (a case report). Indian J Ophthalmol. 1986;34:67-8.

15. Jordan DR, MacDonald H, Noel L, et al. Alveolar soft part sarcoma of the orbit. Ophthalmic Surg. 1995;26:269-70.

16. Chu WC, Howard RG, Roebuck DJ, et al. Periorbital alveolar soft part sarcoma with radiologic features mimicking haemangioma. Med Pediatr Oncol. 2003;41:145-6.

17. Khan AO, Burke MJ. Alveolar soft-part sarcoma of the orbit. J Pediatr Ophthalmol Strabismus. 2004;41:245-6.

18. Chan WM, Liu DT, Lai CK, et al. Soft tissue sarcomas. Case 2. Orbital alveolar soft part sarcoma in a child. J Clin Oncol. 2004;22:2027-9.

19. Morris WR, Padgett DM, Osborn FD, et al. Pathologic quiz case: an orbital mass in a 45-year-old woman. Alveolar soft part sarcoma. Arch Pathol Lab med. 2005;129:534-6.

20. Kim HJ, Wojno T, Grossniklaus HE, et al. Alveolar soft-part sarcoma of the orbit: report of 2 cases with review of the literature. Ophthalmic Plast Reconstr Surg. 2013;29(6):e138-42.

21. Rose AM, Kabiru J, Rose GE. Alveolar soft-part sarcoma of the orbit. Afr J Paediatr Surg. 2011;8:82-4.

22. Hei $Y$, Kang $L$, Yang $X$, et al. Orbital alveolar soft part sarcoma: $A$ report of 8 cases and review of the literature. Oncol Lett. 2018;15(1):304-14.

23. Nava-Castañeda Á, Tovilla-Canales $J$, et al. Alveolar soft part sarcoma of the orbit, a case report of a rare tumor. Nepal J Ophthalmol. 2017;9(18):70-3.

24. Chaudhari PB, Pathy S, Deo SSV, et al. Alveolar soft part sarcoma of orbit: A rare diagnosis. J Egypt Natl Canc Inst. 2017;29(3):167-70.

25. Kumar GK, Nemade H, Krishnamohan, et al. Rare case report of alveolar soft part sarcoma of the orbit. Indian J Surg Oncol. 2017;8(2):234-9.

26. Mulay K, Chougule SS, Honavar SG. Orbital Alveolar Soft Part Sarcoma. Ophthalmology. 2016;123(7):1420

27. Mulay K, Ali MJ, Honavar SG, et al. Orbital alveolar soft-part sarcoma: clinicopathological profiles, management and outcomes. J Cancer Res Ther. 2014; 10(2):294-8.

28. Majumdar K, Saran R, Tyagi I, et al. Cytodiagnosis of alveolar soft part sarcoma: Report of two cases with special emphasis on the first orbital lesion diagnosed by aspiration cytology. J Cytol. 2013;30(1):58-61.

29. Alkatan H, Al-Shedoukhy A, Chaudhry I, Ayoubi A. Orbital alveolar soft part sarcoma: Histopathologic report of two cases. Saudi J Ophthalmol. 2010; 24(2):57-61.

30. Ladanyi M, Lui MY, Antonescu CR, et al. The der (17) t (X; 17)(p11;q25) of human alveolar soft part sarcoma fuses the TFE3 transcription factor gene to ASPL, a novel gene at 17q25. Oncogene. 2001;20:48-57.

31. Hodge JC, Pearce KE, Wang $X$, et al. Molecular cytogenetic analysis for TFE3 rearrangement in Xp11.2 renal cell carcinoma and alveolar soft part sarcoma: validation and clinical experience with 75 cases. Mod Pathol. 2014;27:113-27.

32. Hornick JL, editor. Practical Soft Tissue Pathology: A Diagnostic Approach. Philadelphia, PA: Elsevier Saunders; 2013. p. 178-80.

33. Sherman N, Vavilala M, Pollock R, Romsdahl M, Jaffe N. Radiation therapy for alveolar soft-part sarcoma. Med Pediatr Oncol. 1994;22(6):380-3.

34. Haddock MG, et al. Chemotherapy, irradiation, and surgery for functionpreserving therapy of primary extremity soft tissue sarcomas: initial treatment with ifosfamide, mitomycin, doxorubicin, andcisplatin plus granulocyte macrophage-colony- stimulating factor. Cancer. 2002;94:786-92.

35. Ogura K, Beppu Y, Chuman H, Yoshida A, Yamamoto N, Sumi M, et al. Alveolar soft part sarcoma: a single-center 26-patient case series and review of the literature. Sarcoma. 2012;2012:907179.

36. Grobmyer SR, Maki RG, Demetri GD, et al. Neo-adjuvant chemotherapy for primary high-grade extremity soft tissue sarcoma. Ann Oncol. 2004;15:1667-72.

37. Cordier JF, Bailly C, Tabone E, Cheix F, Brune J, Touraine R, et al. Alveolar soft part sarcoma presenting as asymptomatic pulmonary nodules: report of a case with ultrastructural diagnosis. Thorax. 1985;40:203-4.

38. Portera CA Jr, Ho V, Patel SR, et al. Alveolar soft part sarcoma: clinical course and patterns of metastasis in 70 patients treated at a single institution. Cancer. 2001;91:585-91.

\section{Publisher's Note}

Springer Nature remains neutral with regard to jurisdictional claims in published maps and institutional affiliations.
Ready to submit your research? Choose BMC and benefit from:

- fast, convenient online submission

- thorough peer review by experienced researchers in your field

- rapid publication on acceptance

- support for research data, including large and complex data types

- gold Open Access which fosters wider collaboration and increased citations

- maximum visibility for your research: over $100 \mathrm{M}$ website views per year

At BMC, research is always in progress.

Learn more biomedcentral.com/submissions 